\title{
Metaphors, models and organisational ethics in health care
}

Jennifer McCrickerd Drake University, Des Moines, Iowa, USA

\begin{abstract}
Crucial to discussions in organisational ethics is an evaluation of the metaphors and models we use to understand the organisations we are discussing. I briefly defend this contention and evaluate three possible models: the current corporate model, an orchestrator model which puts hospitals in the same class as malls and airports, and a community model. I argue that the corporate and orchestrator model push to the background some important organisational ethics issues and bias us inappropriately towards certain solutions. Furthermore, I argue that the community model allows these to be more easily brought up. I also respond to the likely challenge that hospitals really are corporations by arguing that this is not relevant to evaluations of the appropriateness of the corporate model.

(Fournal of Medical Ethics 2000;26:340-345)
\end{abstract}

Keywords: Metaphor; model; organisational ethics; health care ethics

"The essential difficulties in social policy have more to do with problem setting than with problem solving, more to do with ways in which we frame the purposes to be achieved than with the selection of optimal means for achieving them ... the framing of problems often depends upon metaphors underlying the stories which generate problem setting and set the direction for problem solving."

Some time ago I attended an organisational ethics miniconference for health care professionals and was surprised to discover that the language and examples used were grounded in the presumption that health care organisations ought to be thought of and act as corporations or businesses - ethical corporations, but corporations none the less. No one at the conference defended this understanding of health care organisations; it was treated as obvious and undeniable. And again, recently a representative from one of the local hospitals was looking for someone who could speak about organisational ethics at a conference; the speakers she was considering were all within business ethics.

Non-profit corporations notwithstanding, the most common psychological association to corporations and business is the idea of profit. Close on the heels of this is the presumption that the corporation is producing a product, has customers and should be "leaner and meaner" to cut costs. And this is just the beginning of a host of associations that follow from identifying something as a "corporation". Despite the obvious corporate atmosphere in heath care, it seemed to me that discussions about ethics would be greatly limited if the corporate nature of heath care organisations was not challenged. With these thoughts in the back of my mind, the above statement from Donald Schön jumped out at me when I read it. He argues that the metaphors we employ are crucial to our framing of issues and this framing deserves considerable attention as it guides both what we consider to be problems and how we will address them. If the way we frame or identify problems largely dictates solutions, we need to spend much more time examining the means by which we frame or identify. We must carefully consider the implications of different alternatives so that the frames we use shape our discussion in an appropriate way.

In this paper I compare three models of health care organisations. I begin with a brief discussion about metaphors and models and then move to discuss viewing hospitals as corporations, as airports or malls, and as communities (because most discussions regarding organisational ethics in health care focus on hospitals, I will be using "hospitals" as the paradigmatic health care organisation. This discussion could quite easily apply to other health care providers as well.) I argue that the first two models bias discussions in organisational ethics in an undesirable way and that serious consideration should be given to viewing health care organisations as communities. Such a model frames organisational issues in a manner that brings to the forefront important issues that can otherwise be lost. In response to the claim that hospitals really are corporations and, thus, we have no choice in the model we use, I argue both that it isn't clear that hospitals are not really communities and that what something really is is not relevant to the question of what model ought to be used to discuss it.

The importance of metaphors and models Illustrating his position that the framing of issues shapes what we take to be potential solutions to problems, Donald Schön uses the example of urban housing. In one cited passage, slums are referred to as blighted, unhealthy, congenitally diseased and requiring an entirely new redesign to root out the cause of the cancer. In another, slums are viewed as natural communities sharing much with working class communities across cultures and history. In this second passage not all slums are viewed as 
problematic deviations from the healthy, but are seen as naturally occurring and providing positive aspects for their inhabitants (stability, high level of social interaction). Schön argues that each perspective on slums, being the result of disease or nature, has dramatically affected urban public policy. One fostered a commitment to redesigning communities, purging them of anything that was diseased, the other to altering communities only as necessary with a stress on minimising relocation of community members as much as possible.

As is clear from Schön's example, when using a metaphor it is the immediate associations we have that are important, not necessarily the legitimacy of the associations. Each of the three models I will be discussing could reasonably be used to understand and guide health care organisations. Additionally, clear boundaries do not exist between all of these models. Many corporations can be viewed as communities; likewise communities can be understood as being corporations - many people today believe that good corporate leaders will make good community leaders. While this is true, what is important for the purposes of this discussion is not the literal accuracy of the metaphor - that is not the purpose of metaphor-but the effect of different metaphors with regard to what is brought to the forefront of discussion and what is pushed into the background. Even if the metaphor conflicts with what the object of the metaphor really is is not important. For example, few families are literally military units, but if someone says "My family wasn't really a family it was a battalion," we not only know what is meant but we do not correct the speaker's use of "family" or "battalion". We recognise that for some purposes some families are not appropriately characterised as "families" and might be better characterised in other ways.

\section{Cowboys and shepherds}

Returning to the example of "slums" being either naturally occurring or diseased, the literal distinction is easily rendered untenable by pointing out that many diseases are naturally occurring. In this same vein, our assumption that natural is good could be challenged. But the force of the metaphor comes not in drawing out a one-to-one correspondence of what associations ought to be if we carefully and critically thought through the metaphorical terms, but, rather, in the associations, whether or not legitimate, that we do have as people who share a common culture. Catherine Elgin offers the comparisons of cowboys and shepherds to illustrate this point. If I describe someone as a "cowboy", particular qualities are assumed for that person. Describing that same person as a "shepherd" would conjure different personality traits. Depending on the metaphor I use, a different sense of the individual is suggested, although cowboys and shepherds do pretty much the same sort of work There is nothing in the reality of the task that would lead us to think that they have different personalities, but the metaphors conjure up different images. ${ }^{2}$ Or, consider the associations we have with the word "wolf". Most of our associations are likely the result of fairy tales and cartoons, having little to do with the reality of this extremely shy, packoriented animal. However, the true nature of the animal is unlikely to make someone who hears others calling him a wolf feel much better. The associations we make may not be the most informed but they are the associations that shape our thoughts and we can assess the appropriateness of the metaphor by assessing the associations that accompany it.

Before I move on to begin such an assessment, let me take a moment to draw the connection between metaphors and models. Other theorists have made the connection by describing models as structural metaphors or as a means of more systematically and comprehensively drawing associations and connections than metaphors do. ${ }^{3}$ Most helpful for me has been Max Black's discussion of what others have called "submerged models" or "root metaphors" and what Black labels "archetypes". Black cites a helpful passage from Stephen Pepper's discussion of "root metaphors" and afterward explains his conception of archetype. Pepper says: "The method in principle seems to be this: a man desiring to understand the world looks about for a clue to its comprehension. He pitches upon some area of commonsense fact and tries if he cannot understand other areas in terms of this one. The original area becomes then his basic analogy or root metaphor... . In terms of these categories [of the original area] he proceeds to study all other areas of fact... . He undertakes to interpret all facts in terms of these categories." " Black's conception of archetype mirrors this idea. "By an archetype I mean a systematic repertoire of ideas by means of which a given thinker describes, by analogical extension, some domain to which those ideas do not immediately and literally apply."

Despite the fact that Black does not use the term "metaphor" here, it is clear that he believes that the adoption of such a repertoire of ideas will shape theoretical discussions in the same way that metaphors shape the discussions of which they are a part. For the purposes of this paper, I will not use "archetype" or "root metaphor", but instead will use "model" for this seems most consistent with common usage. While at a theoretical level one could challenge and push all of these terms, looking for more finessed understandings, I believe that for the purposes of my discussion, identifying and dealing with such challenges is unnecessary. What is most important is the idea that the models we use have a metaphorical quality insofar as they influence conversations in the same way metaphors do.

Having explained the importance of metaphors and models, I now move on to discuss three potential models for health care organisations: the corporate/business model, the orchestrator (airport/mall) model and the community model.

\section{Hospital as corporation/business}

In his paper, "From clinical ethics to organisational ethics: the second stage of the evolution of bioeth- 
ics," Robert Lyman Potter calls for an expansion of health care ethics into organisational ethics, saying that such an expansion is a "logical and practical step toward achieving the patient-oriented goals of clinical ethics and is a turn to the broad ecological version of bioethics" ${ }^{6}$ As Potter discusses this "turn to the broad ecological version of bioethics", he explains that moving from clinical concerns to ecological concerns, we must move through concentric rings of ever expanding concerns-clinical, corporate, social policy, international human rights, and, finally, the ecological. Ideally, we would work on all five rings at the same time, but in reality we must focus on the corporate sphere now. What I find interesting in this work is that while Potter is advocating an ultimate shift to an ecological paradigm, his discussion of hospitals centres on their corporate nature. In fact, he soon equates "organisational ethics" with "corporate health care ethics" and says that one of the primary ethical questions facing health care organisations is: "How do we create an ethical corporate culture that makes ethics as important for heath care decisions as clinical data, financial concerns, and legal issues?"7 The corporate culture is not questioned; it is the accepted circumstance within which we must discuss ethics.

\section{Business image}

I am not suggesting that those who discuss organisational ethics and adopt a corporate model of health care institutions are making egregious errors. Of course, there is an obvious corporate aspect of health care providers these days. Examples of this being emphasised in health care can be seen in the reference to top administrators as chief executive officers, chief financial officers, etc ${ }^{8}$ or in the "budgetary concerns" that frequently drive decision making. Also perpetuating this business image is the increase of commercial advertising for hospitals or hospital programmes. But how does focusing on and further solidifying the view of hospitals as corporations in our discussions of ethics shape our discussion of organisational ethics and is there a worthwhile alternative? What are the associations we make when an organisation is viewed as a corporation and how will this shape our ethical discussions with regard to the organisation? What will appear "natural" and what will appear out of place?

To begin with, a corporation is concerned with money and so customer happiness is a priority, thus there is an emphasis on customer satisfaction. In health care organisations this translates to a focus on patient satisfaction (or, more problematically, insurer satisfaction) rather than an emphasis on patient care, although it is to be hoped that the two intersect. The association of health as a commodity or product follows easily from an understanding of health care organisations being corporations as does the understanding of the health care professional/patient relationship being a producer/customer or contractor/ client relationship. Additionally, corporations are viewed as primarily self-interested; the extent to which a corporation cares about others is the extent to which caring about others will be in the corporation's best interest.

All these associations are quite different from our usual association of health as something beyond product, as something necessary for a quality life, akin to safety from harm or access to education. These associations also conflict with our traditional conception of the provision of medical care as inspired by caring and being something close to an art. Hospitals are not taking care of patients with this model; they are meeting a market need. Furthermore, this is a dramatic shift away from viewing people (whether patients, health care providers or support staff) as important in and of themselves to a view of people as being important insofar as they can help the corporation meet its financial goals. Certainly, people who operate under the corporate model are not likely intending or delighting in these associations, but whether deliberate or not these associations will permeate and shape discussions in and about the organisation. I believe one of the clearer effects will be in the area of ethics.

The ethical responsibilities when producing a product and dealing with customers revolve around being honest about one's product and meeting the customers' desires in as truthful a manner as possible, although there is always the caveat of "buyer beware". This translates into primary ethical concerns being informed consent and autonomous decision making. This is not to say that these are not important issues; clearly they are. Nor is this to say that the corporate model excludes other ethical issues, only that they are not as obvious. Issues such as the appropriate attitude health care professionals and organisations ought to have towards patients, responsibilities that a health care organisation might have towards the community of which it is a part, the level of commitment health care organisations ought to have to programmes that do not bring in money but only use it (for example, chaplaincy programmes and social services) or the treatment of hospital employees, will not present themselves as obvious areas of ethical concern when the health care organisation is considered a corporation. Of course, all of these can be, and are, brought up within a corporate conception, but my contention is that by viewing hospitals primarily as corporations, we centre the conversation on those things that are immediately associated with corporations. While other conversations are possible, they are not easily introduced, nor will they fit neatly into the framework.

With the corporate/business model, the primary concern of the institution is economic, with codes of ethics acting as a means to prevent unethical means of achieving the economic goals.

\section{Hospital as orchestrator (á là airport or mall)}

Christopher Press has recently suggested that hospitals should view themselves as orchestrators of providers not as providers themselves. ${ }^{9}$ Instead of focusing on the corporate quality of the current model, he argues that today's understanding of health care institutions is modelled after a factory 
paradigm - that the institution should provide the whole package for the consumer at as low a cost as possible. Instead of understanding hospitals to be manufacturers of health, Press suggests that we regard hospitals as being orchestrators providing "a functional, economical infrastructure".$^{10}$ In the same way that airports and malls provide infrastructure for airlines and stores, hospitals would provide infrastructures for specialised, independently run groups. What would be the implications of such a vision of health care organisations?

Most associations with malls and airports are fairly negative - a primary association being that of an impersonal space. But if we focus on the idea of hospitals being orchestrators instead of hospitals being in the same category as malls or airports, different associations may come to bear. With an orchestrator model, each specialty is fairly autonomous. This, Press argues, is an asset of the model "Each enterprise would serve its constituents and earn returns in proportion to its risks." ${ }^{11}$ Specialties and sub-specialties would form alliances with each other when mutually beneficial and, as with the more general corporate model, supply and demand as well as cost effectiveness and risk-benefit outcomes would determine who remains a provider within the hospital and who does not.

The interconnectedness of health care makes the model of orchestration an odd one. Unlike malls and airports where adjoining merchants/providers are, at best, disinterested in each other's success, the services provided in a hospital cannot be easily divided into individual compartments. Furthermore, while some services generate little or no income, these services may be quite valuable for the patient. But if each service provider must generate its own income, these services won't be provided in a hospital acting as an orchestrator. If we understand the hospital to be an orchestrator, organisational issues will not easily be identified or dealt with at the hospital level but instead will be pushed to the level of the individual specialty where the corporate model will likely prevail. Such a move will discourage discussions regarding the ethical responsibility of the whole to provide social services, spiritual support and the like. This consequence is not surprising, as Press's concern with the current model of health care organisations is that it does not lead to cost-efficient hospitals. His move to hospitals as orchestrators may solve this problem, but the larger cost is that it will be more difficult to discuss the possibility of an obligation to provide services that do not generate income, but do provide a needed service and will merely shift other organisational ethical issues to a different market-driven body.

Of course, an advocate of this model could respond by pointing out that malls and airports have parking lots, security guards, custodial staffs and the like which do not generate income, yet certainly exist. So, social services, chaplaincy programmes, etc could be viewed as analogous to these services. Furthermore, the specialties which make use of the coordination of the hospitals need not pattern themselves after corporations or even small businesses. The orchestration model is merely calling upon hospitals to stop viewing themselves as providers of health and instead see themselves as providers of infrastructures for those who actually can provide health. The model implies nothing about how we view health providers.

Of course the orchestrator model does not prevent the specialties from thinking of themselves as other than corporations or small businesses, nor does it eliminate the possibility of discussing the provision of non-income generating services, but it does make these more difficult in virtue of the framing provided. While a group taking advantage of the coordinating efforts of the hospital could view itself as something other than a small business or corporation, the model, with its emphasis on cost-efficiency, will not encourage this. Likewise, while non-income generating services could become a main topic of conversation, when hospitals are orchestrators this topic will not likely arise. The emphasis is likely to be put on providing the infrastructure needed for the independent specialties, not additional services that the independent specialties are unlikely to provide.

\section{Hospital as community}

A last model to examine is that of hospital as a community. While surely a romantic vision, any collection of individuals characterised as a "community" will be presumed to be populated with people who care about one another, people who prioritise relationships. Associating health care institutions with communities would put relationships between the individuals in the community as well as community wellbeing at the forefront of conversations. In a society predisposed toward democratic government, such a model would also shift hospitals away from the presumption that decisions be made at the top and then communicated to those the decisions will affect. In the centre of this model are the people and, importantly, all people-from physicians and patients, who are frequent subjects in health care ethics discussions, to other health care professionals and hospital staff who are not.

While the community model does not ignore the issues that other models put in the forefront, it does shift them from the centre. Communities must still be concerned with finances and sustainability, but with a community instead of corporate model, the concern with these matters will more easily and clearly be couched in a larger concern for the people in the community. Also, in the same way that Press's model of hospital as orchestrator encourages health care institutions not to view themselves as trying to provide everything, but instead providing an infrastructure, the model of a community also encourages a view of groups working together instead of a monolithic entity controlling everything. However, the autonomy of the groups is more moderate than that conjured with the orchestrator view, for in a community individual shops or families, etc, must interact 
with, consider and possibly have responsibilities to others in the community.

Two examples of where a community model has had some success, though it may be waning with the growing influence of the corporate model, is in higher education and some religious denominations. Each of these, like health care institutions, are providing services for people in need. Likewise each has economic constraints and potentially large constituencies. But in both education and religion significant emphasis is put on the individuals of the constituencies, whether employees or recipients of care, being at the centre of concern.

The largest difficulty with the community model may also be one of its major strengths-it does not provide an easy resolution for the potential conflict between the good of the community and the good of the individual. This mirrors at least one dynamic in hospitals that is lost in other models' prejudice towards the position that sacrifice will come from individuals, which makes discussion of an alternative difficult. Currently, within the corporate model, employee reductions, longer hours, etc, are easily justified by an appeal to the bottom line and can be legitimately challenged only when the changes negatively affect the consumers. With a community model, other factors, such as the appropriate treatment of employees, can be more easily brought up and viewed as more legitimate.

\section{Response to possible objections}

At this point an objection might be made that regardless of the associations that accompany different metaphors or models the fact of the matter is that health care organisations simply are corporations. Because they are businesses, hospitals must be identified as such and until health care organisations are no longer corporations, there is no point in brushing aside this fact.

While clearly many health care organisations are legally corporations (that is, are incorporated, etc), it is not at all clear that discussions of health care organisations must follow a corporate model. First of all, the legal status of hospitals has not changed dramatically in the last 50 years, yet the corporate view of hospitals is fairly new. This suggests that regardless of the legal status of hospitals, focusing on their corporate nature is not necessary. Secondly, while it is true that health care organisations are corporations; they are also communities. Focusing on the community aspect of health care organisations is as justified by literal truth as focusing on the corporate. Additionally, many entities are incorporated but do not view themselves as corporations. Schools, communities, individuals can and do incorporate themselves; they are corporations. But each of these entities can meaningfully be viewed as other than a corporation.

Lastly, the literal identity of something is not relevant to what model or metaphor ought to be used. Metaphors and models function to draw our attention to certain characteristics of the object in question. The associations accompanying the model or metaphor, not the literal meaning, shape discus- sions. The legal, literal manner in which health care organisations are corporations is unrelated to their metaphorical identification as corporations. In a sense, the literal, legal corporate nature is moot. Being incorporated has a specific legal meaning which varies from the associations that come along with "corporation".

\section{'Family'}

Consider, for example, the different uses of "family". When investigating the possibility of genetic disease it is perfectly accurate to discuss the family connections of relatives who have never met one another. For the purpose of genetics "family" has a very specific meaning and to say that one's adoptive parent is not family is not particularly controversial. However, in other contexts, "family" will not be appropriate even when discussing people who are biologically related-"I know she's my grandmother, but having never met her, she was never really part of my family". And while a lifelong friend may not literally be family, excluding him or her from "family only" events may be unthinkable. Likewise, it is appropriate to discuss the corporate nature of health care institutions while discussing corporate law, but outside of corporate law discussions and particularly in discussions about ethics, the corporate identity may be more harmful than helpful.

Examining these different models and the implications for the way we think about organisations, one recognises that the process of choosing a model is not a matter of carefully analyzing all possibilities to determine which is the right model any more than choosing a child's name is a matter, merely, of trying out names until one happens upon the name that the child was meant to have. With names we consider, among other things, possible nicknames (chosen or inflicted), the associations family members will have, the impressions people will have of our child when they first hear the name, and the child's future reaction to the name. Much in the same way, with a model or metaphor we consider the implications of our choice, the fit it may have, how well it serves our purposes, what issues will be viewed as central and which will be viewed as diversions, worthwhile diversions when circumstances and time permit, but diversions none the less. In both types of decisions, it is obviously true that the concerns we have going in are going to shape the ultimate decision we make. This does not, however, mean that we are simply begging the question; inevitably the values called upon to make the decisions will be scrutinised. Models can be challenged and defended with reference to values and purposes which, likewise, can be challenged and defended.

The recognition that the models we use shape our discussions by providing a frame through which we view is crucial in ethics. Given that any model or metaphor used is going to make some questions less likely to arise or even incomprehensible and will make some practices prima facie acceptable or unacceptable, we need carefully and deliberately to choose our models. 
The consequences for the discussions about organisational ethics of adopting the corporate model are serious enough to encourage examination of alternatives. On the top of the list of alternative models I would put the community model. Of course, communities must also be concerned with not going bankrupt, so economic issues would not be entirely pushed aside, but no longer would the economic relationships and concerns be viewed as the primary concern, nor would they have the most power in shaping our perspective elsewhere.

\section{Acknowledgement}

Thanks to two fournal of Medical Ethics reviewers for suggestions for improvement.

Fennifer McCrickerd, PhD, is Associate Professor, Philosophy Department, Drake University, Des Moines, Iowa, USA.

\section{References and notes}

1 Schön D. Generative metaphor: a perspective on problemsetting in social policy. In: Ortony A, ed. Metaphor and thought. Cambridge: Cambridge University Press, 1979: 255.

2 Elgin C. Considered judgment. Princeton, NJ: Princeton University Press, 1996: 188-9.

3 Childress J. Who should decide? New York, NY: Oxford University Press, 1982; Childress J. Practical reasoning in bioethics. Bloomington, IN: Indiana University Press, 1997.

4 Pepper S. World hypotheses. Berkeley and Los Angeles: University of California Press, 1942:91.

5 Black M. Metaphor and model. Ithaca, NY: Cornell University Press, 1962: 241.

6 Potter R. From clinical ethics to organisational ethics: the second stage of the evolution of bioethics. Bioethics Forum 1996;12:3-12.

7 See reference 6: 8 .

8 Thanks to Robin Downie for noting this blatant example.

9 Press C. The hospital as airport: a new model for health care.

Health Forum fournal 1999; 42:50-4.

10 See reference 9: 52 .

11 See reference 9:53. 